\title{
¿ES $L A$ INTUICIÓN LÓGICAMENTE ANTERIOR A LA INTUICIÓN INTELECTUAL EN KANT? ${ }^{1}$
}

\section{IS THE INTUITION LOGICALLY PRIOR TO INTELLECTUAL INTUITION IN KANT?}

\author{
Luis Placencia \\ Universidad de Chile \\ Ignacio Carrera Pinto 1025 \\ Santiago, Chile \\ luisplacencia@gmail.com
}

\begin{abstract}
Resumen
En este artículo pretendo mostrar una oscuridad relativa al concepto de intuición que se conecta con otro aspecto oscuro y comparativamente menos tratado de su obra, sc., sus afirmaciones sobre la intuición intelectual. Según la manera más habitual de
\end{abstract}

$1 \quad$ Este artículo es resultado del proyecto Fondecyt 1151001, "Autoconocimiento y autoconciencia en Kant. Sobre las formas del conocimiento y del saber de sí en la filosofía crítica". Versiones previas de este trabajo fueron presentadas a diferentes audiencias en Ciudad de México, Temuco y Talca. Por sus comentarios y sugerencias agradezco a todos quienes participaron de la discusión en esas instancias, especialmente a Luis Eduardo Hoyos, Efraín Lazos, Roberto Rodríguez Aramayo, Pedro Stepanenko y Ezequiel Zerbudis. También he podido aprovechar para este trabajo una serie de observaciones de Francisco Abalo y Javier Fuentes. Por la ayuda en la corrección del manuscrito agradezco a Matías von dem Bussche. 
considerar la relación entre estos términos, Kant tematizaría una noción de intuición -lo que en el título llamo la intuición-que constituiría a su vez un género común de al menos dos especies, a saber, la intuición sensible y la intuición intelectual. Con ello, quedaría establecida la anterioridad lógica de la noción de la intuición, esto es, el hecho de que las nociones de intuición sensible e intuición intelectual serían definibles solo por recurso a la intuición y no viceversa. Un examen riguroso del asunto, creo, mostrará como resultado que este prejuicio es altamente cuestionable. Es este último el punto que me interesa establecer en este texto.

Palabras clave: Kant, intuición, intuición intelectual, anterioridad lógica, critica de la razón pura.

\begin{abstract}
In this paper I explore the lack of clarity concerning the concept of "intuition" that is connected with another obscure aspect of Kant's work that has received comparatively less attention, his claims about "intellectual intuition". According to the typical understanding of the relationship between these terms, Kant would thematize a notion of "intuition" -what I call the intuition- which would constitute in turn a common genus of at least two species, "sensible intuition" and "intellectual intuition". This understanding establishes the logical priority of the notion of intuition, that is, that the notions of "sensible intuition" and "intellectual intuition" would be definable only by recourse to the intuition and not vice versa. I show in this paper that this prejudice is highly questionable.
\end{abstract}

Keywords: Kant, Intuition, Intellectual Intuition, Logical Priority, Critique of Pure Reason

Recibido: 06/03/2019

Aceptado: 23/07/2019 


\section{INTRODUCCIÓN}

No parece ser una afirmación arriesgada el sostener que la noción de intuición juega un papel fundamental en la filosofía de Kant, al menos tal y como ella adquiere forma a partir de la Crítica de la razón pura (en adelante $K r V$ ) o incluso antes en la Disertación sobre la forma y principios del mundo sensible e inteligible. En efecto, son muchas las tesis kantianas que, al menos a primera vista, solo pueden ser comprendidas a cabalidad bajo el supuesto de que se haya captado de manera adecuada el significado de la expresión antedicha. Lo anterior parece ser, por ejemplo, lo que ocurre con la conocida idea kantiana según la cual, para que haya conocimiento, deben concurrir intuiciones y conceptos, tesis que es expresada del siguiente modo en un pasaje crucial de la introducción a la "Lógica trascendental":

Si queremos llamar sensibilidad a la receptividad de nuestro ánimo para recibir representaciones en cuanto él es afectado de alguna manera, entonces es, por el contrario, el entendimiento la facultad de producir representaciones por uno mismo o espontaneidad del conocimiento. Nuestra naturaleza es tal que la intuición no puede nunca sino ser sensible, esto es, solo contiene el modo en que somos afectados por los objetos. Por el contrario, el entendimiento es la facultad de pensar el objeto de la intuición sensible. Ninguna de estas propiedades ha de ser preferida a la otra. Sin la sensibilidad no nos serían dados objetos y sin el entendimiento no podrían ser pensados. Pensamientos sin contenido son vacíos, intuiciones sin conceptos son ciegas [...] Solo del hecho de que ambas facultades se unifiquen puede surgir conocimiento. Por eso no se puede mezclar su contribución, sino que hay buenas razones para abstraer y diferenciar la una de la otra. (A 51/B 75) ${ }^{2}$

Como es habitual entre los especialistas, cito las obras de Kant recurriendo a la edición comenzada por la Academia Prusiana de Ciencias y continuada desde 1966 por la Academia de Ciencias de Gotinga, que indico con la sigla AA seguida del volumen, página y línea de la misma (v. gr., AA 20266 14-18). La excepción a esta práctica la constituye la $\mathrm{KrV}$ que se cita remitiendo a la paginación de la primera y segunda ediciones, indicadas como A y B respectivamente (v. gr., A 51/B 75). En el caso de Fichte, remito a la edición de la obra completa editada por Academia Bávara de 
El pasaje, que resume de modo notable una parte esencial de las enseñanzas de la $K r V$, no solo destaca la famosa tesis de la necesaria colaboración de intuiciones y conceptos para que haya conocimiento (tesis que ha devenido un asunto de interés no solo para los especialistas en Kant, sino incluso de modo creciente para scholars de otros ámbitos a partir de la publicación de Mind and World de John McDowell), sino que además pone de relieve el hecho de que es necesaria en virtud del carácter heterogéneo de las facultades de las cuales este tipo de representaciones son actos, a saber, sensibilidad y entendimiento, toda vez que responden a formas de representar completamente diferentes. Mientras el entendimiento es una facultad activa de conocimiento, basada en la espontaneidad, la sensibilidad es una facultad pasiva de conocimientos, basada en la receptividad. La tesis que está detrás de la posición de Kant parece ser, entonces, que para que haya conocimiento es necesario el concurso de estos dos rendimientos: intuiciones y conceptos. Así las cosas, intuiciones y conceptos serían condiciones sine quibus non para conocer, pues cada una proveería elementos necesarios para el conocimiento no provistos por la otra. Pero al ser la intuición humana sensible (A 19/B 33), sería entonces para nosotros imposible conocer aquello que no nos es dado a la sensibilidad (B 165). Como se sabe, esta es una de las tesis fundamentales de la filosofía crítica, que está a la base de las tesis limitativas respecto de la posibilidad de la metafísica en la $\mathrm{KrV}$. Lo anterior, sin embargo, nos da cuenta solamente de una de las razones por las cuales resultaría esencial comprender el significado del término intuición en Kant, al menos para captar con precisión una de las tesis centrales de su obra fundamental. En efecto, sin comprender el significado del término intuición, parece ser que no seríamos capaces de comprender cuál es el tipo de acto al cual parece estar limitado el conocimiento humano. A lo anterior pueden sumarse, sin embargo, muchas otras ideas claramente sostenidas por Kant que parecen también depender de la comprensión adecuada del significado de intuición. De ellas podemos mencionar, por ejemplo, el hecho

Ciencias, indicando el tomo, subtomo y página (v. gr., I 4 278). Por último, cito los textos de Aristóteles, Porfirio y Proclo siguiendo también las convenciones clásicas entre especialistas. Todas las traducciones son mías. 
de que tal noción parece ser fundamental para captar tanto la concepción que tiene Kant del modo en que opera la geometría (A 25/B 40-1) como la de la misma posibilidad de los juicios sintéticos a priori (B 73), cuestión que sabemos, constituye "la tarea propia de la razón pura" (B 19). Junto con ello, el significado del término intuición parece ser fundamental para comprender asuntos que, aunque en apariencia son de menor relevancia para las enseńanzas de la $K r V$ que los indicados anteriormente, forman parte esencial de la filosofía crítica de Kant. Un ejemplo de lo anterior es el tópico de la posibilidad del conocimiento de sí mismo (B 66-9) que Kant aborda con creciente insistencia ${ }^{3}$. Como es bien sabido, este problema es el que abre la puerta en la tradición poskantiana conocida como idealismo alemán a la admisión de la posibilidad -descartada por Kant- de una intuición intelectual, tal como se puede apreciar en la obra temprana de J. G. Fichte (I 4 278) $)^{4}$.

Con todo, y más allá de la relevancia del significado de intuición para la comprensión de la $K r V$ en particular y de la filosofía crítica en general, no parece haber acuerdo entre los intérpretes de Kant sobre el significado que ha de atribuírsele a esta expresión, especialmente habida cuenta las oscuridades y dificultades que parece entrañar el modo en que Kant caracterizó el término. De hecho, en la literatura reciente se ha sugerido que Kant emplea el término con una significación doble (Falkenstein 70; Grüne 30-1; Sellars 7) o incluso triple (Allison 82). Por otra parte, las ambigüedades que el término parece entrañar de suyo suelen ser destacadas en la literatura (Ewing 17-8; Paton 93-8) así como las que el uso kantiano del mismo trae consigo, haya sido Kant o no consciente de ello (Allison 82). Sin embargo, no es este aspecto del problema el que me interesa tratar aquí. En este texto, más bien, pretendo mostrar una oscuridad relativa al concepto de intuición que se conecta con otro aspecto oscuro y comparativamente menos tratado

Piénsese en los agregados de la segunda edición -que abordan este problema con mucho mayor detalle que en la primera- como el ya indicado pasaje de B 66-9; B 157-9; B 274-9; B 376-436.

4 La historia de este concepto en esa tradición ha sido estudiada, a partir de Kant, en el muy informativo libro de X. Tilliette (1995). 
de su obra, a saber, sus afirmaciones sobre la intuición intelectual. Según la manera más habitual de considerar la relación entre estos términos, Kant tematizaría una noción de intuición -lo que en el título de este trabajo llamo la intuición- que constituiría a su vez un género común de al menos dos especies: la intuición sensible y la intuición intelectual. Con ello, quedaría establecida la anterioridad lógica de la noción de la intuición, esto es, el hecho de que las nociones de intuición sensible e intuición intelectual serían definibles solo por recurso a la intuición y no viceversa ${ }^{5}$. Esta interpretación parece haber sido importante para autores muy relevantes en la historia de la interpretación de una noción tan básica como la que así se discute (v. gr. Hintikka, cuyas ideas serán discutidas más adelante). Un examen riguroso del asunto, creo, mostrará como resultado que este prejuicio es altamente cuestionable. Es este último punto el que me interesa establecer en este trabajo.

Quisiera desactivar, antes de continuar, una objeción que me parece relativamente obvia. Como se ha dicho con anterioridad, parte de la literatura ha puesto de relieve que Kant haría un empleo doble o incluso triple de la noción de intuición. En efecto, mientras Kant destacaría en un conocido pasaje -que abordaré con más detalle a continuación (A 320/B 376-7) - que las intuiciones serían representaciones con conciencia y que estarían referidas a objetos, existirían otros pasajes, según algunos de los comentadores mencionados, en que estos requerimientos no estarían ligados a la noción de intuición (v. gr. A 51/B 75) . Es de destacar que la mayor parte de estos autores identifican un concepto propio (Grüne) o típico (Allison) de intuición, del cual eventuales otros usos impropios se desviarían. Más allá de la corrección de la lectura de Allison, Falkenstein, Grüne y Sellars, me interesa poner de relieve que el problema sobre el que quiero llamar la

5 Entiendo, en efecto, por anterioridad o prioridad lógica el tipo de relación de anteroposterioridad entre dos ítems que ya Aristóteles tematiza en Metafísica (1028a 34-6), según la cual entre dos ítems $a$ y $b$, $a$ es anterior lógicamente a $b$, si y solamente si $a$ puede ser definido sin recurrir a $b$, pero no viceversa, esto es, si la definición de $b$ incluye a $a$. Para un estudio de esta noción de prioridad en la obra aristotélica, véase Vigo (45-6, 62).

6 Para una lista complementaria de pasajes, se puede ver el trabajo de Grüne (31). 
atención aquí es independiente del de la multiplicidad de significados del término. En efecto, en el punto que a mí me concierne se trata más bien de la prioridad lógica que tendría cualquiera de estos significados que sea tomado como primario sobre las caracterización de la noción de intuición intelectual. Según lo que quiero establecer en este texto, hay buenas razones para pensar que tal prioridad no existe, de suerte que la intuición intelectual no sería una especie de la intuición sin más tal como la trata Kant.

\section{2. ¿Qué es una intuición? Algunas dificultades}

Como se indicó, la relevancia que posee el concepto de intuición para Kant parece contrastar con la poca claridad que arrojan sus textos a la hora de dar cuenta de qué es exactamente lo que mienta. Esta afirmación podría producir perplejidad en alguien que conozca las fuentes. En efecto, Kant señala en reiteradas ocasiones que la intuición (Anschauung) es un conocimiento inmediato y singular, diferenciándose así de los conceptos (Begriffe) que tienen por notas características el ser mediatos y generales. Tal caracterización aparece en diversos textos, al menos a primera vista, como una definición y es tomada de ese mismo modo por una parte considerable de la literatura ${ }^{7}$. Un conocido pasaje de la $K r V$ que cito parcialmente a continuación parece respaldar esta manera de entender la noción de intuición:

He aquí una escala (Stufenleiter) de las representaciones: El género es la representación sin más (raepresentatio). Bajo él están las representaciones con conciencia (perceptio). Una percepción que solo se refiere al sujeto como modificación de su estado es una sensación (Empfindung) (sensatio), una percepción objetiva es conocimiento. Este es o bien intuición o bien concepto

Piénsese, por ejemplo, en el modo en que Friebe abre el artículo "Intuición” en el KantLexikon recientemente publicado bajo la edición de Willaschek, Stolzenberg, Mohr y Bacin: "mientras los conceptos son representaciones generales que corresponden a una multiplicidad de objetos posibles y se refieren solo mediatamente (por medio de sus notas) a objetos, las intuiciones constituyen una referencia inmediata a objetos singulares” (100). Véase también Grüne (35). 
(intuitus vel conceptus). La primera se refiere inmediatamente al objeto y es singular, la segunda mediatamente, por medio de una nota que puede ser común a más cosas. El concepto es o bien empírico o bien puro, y el concepto puro, en cuanto tiene su origen solamente en el entendimiento, se llama noción. Un concepto a partir de nociones que sobresalen a la posibilidad de la experiencia, es la idea o el concepto de la razón. (A 320/B 376-7)

Este fragmento, que E. Lazos ha dado en llamar recientemente pasaje clasificatorio, parece sugerir, en consecuencia con lo anteriormente dicho, una definición de intuición como representación inmediata y singular. Otro tanto ocurre con otros pasajes similares que se pueden hallar principalmente en el legado manuscrito de Kant. Así, por ejemplo, indica Kant en los Progresos "toda experiencia consiste en una intuición, a saber, una representación inmediata y singular" (AA 20266 14-8) o en las "Reflexiones de metafísica": "la intuición es una referencia inmediata de la capacidad representativa a un objeto singular" (AA 18282 31-2 R 5643). Con todo, el pasaje clasificatorio resulta crucial para la lectura según la cual Kant aquí definiría la intuición (sea el único concepto que haya de ella o su sentido propio o focal) en tanto se trata de un pasaje de la obra publicada. Me concentraré así, por ahora, en este fragmento.

De acuerdo con la lectura habitual del pasaje clasificatorio, la caracterización de la intuición que ahí tiene lugar ocurriría en el marco de una taxonomía de representaciones (Smit 235). Esta taxonomía suele ser comprendida como una especie de árbol de Porfirio de las representaciones, esto es, de una clasificación en géneros y especies, que incluye a la representación como género supremo, bajo el cual caería la percepción y así especies de este género identificadas a partir de la posesión de propiedades contradictorias $^{8}$. Todos los elementos identificados habrían de ser entonces tales que contuvieran bajo una definición unívoca al género del cual son especies. Así, bajo la noción de percepción caerían como especies la

$8 \quad$ Porfirio presenta el tipo de relación de orden que llamamos árbol de Porfirio en su Isagogé (I 6). 
sensación y el conocimiento, que serían por ello formas de percepción. Estos géneros y especies, entonces, habrían de identificar clases distintas de representaciones y, por ello, entidades distintas entre sí. Así, por ejemplo, bajo el género conocimiento caerían las especies intuición y concepto, que serían conocimientos (y no sensaciones), de modo que toda sensación no sería conocimiento y todo conocimiento no sería sensación. Esta lectura que da cuenta del pasaje en comento como presentando un árbol de Porfirio (que llamaré lectura del árbol) es asumida de modo más o menos explícito por muchos autores que dan cuenta de este pasaje apelando al repertorio de géneros y especies (Grüne 35 y ss.; Lazos 24; Smit 244). En lo que sigue intentaré sostener que esta lectura es dudosa y con ello aportaré una primera razón para arrojar dudas sobre la idea de que la intuición sería lógicamente anterior a las expresiones intuición sensible e intuición intelectual. En efecto, si es plausible la lectura del árbol, se hace además plausible la idea de que Kant define un género llamado intuición del cual la intuición intelectual sería especie. Por el contrario, si esta lectura no lo es, el supuesto que pretendo poner en cuestión en este texto pierde plausibilidad.

Comencemos, de todos modos, comprendiendo por qué parece ser razonable la lectura del árbol. Esta parece fundarse en razones textuales sólidas, como queda claro ya por el hecho de que el mismo Kant llama género (Gattung) a la noción de representación sin más (Vorstellung überhaupt). Sin embargo, hay también buenas razones para poner en duda esta forma de interpretar el pasaje. Una de ellas, es que, como se deja ver de modo claro a partir del contexto en que es introducido, no tiene por función establecer una clasificación de las representaciones, sino más bien presentar los conceptos que Kant llama ideas. Recuérdese que el pasaje corresponde a la primera sección del primer libro de la Dialéctica trascendental, en concreto al título "De las ideas sin más" (Von den Ideen überhaupt). Aunque esta función es compatible con la presentación de una clasificación o taxonomía de las representaciones e incluso puede suponerla, no parece, por otro lado, exigirla de suyo. A lo anterior se suma, que si se toma la clasificación anterior como una propia de un árbol de Porfirio, surgen claras dudas respecto de la consistencia entre las afirmaciones de la pretendida clasificación y otros textos de la misma 
$K r V$. Así, por ejemplo, mientras la sensación cae en el pasaje clasificatorio del lado de la percepción que no se refiere al objeto y que las intuiciones y los conceptos serían percepciones objetivas, la caracterización que da el mismo Kant de la intuición empírica en A 20/B 34 parece no identificar la sensación con un tipo de representación distinto de la intuición, al menos de la intuición empírica, sino más bien como una parte componente y, por cierto, necesaria de ella. En efecto, en el pasaje en cuestión sostiene Kant lo siguiente: "el efecto de un objeto en la capacidad representativa en cuanto somos afectados por él es la sensación. Aquella intuición que se refiere al objeto por medio de la sensación se llama intuición empírica" (A 20/B 34). Este pasaje sostiene de modo claro que la intuición empírica refiere a objetos por medio de su contenido, a saber, la sensación, de manera tal que la intuición empírica no podría caer meramente del lado de aquello que no es sensación, ni tampoco la sensación ser considerada sin más como carente de referencia a objetos y como constituyendo una representación diferente de la intuición. De este modo, me parece claro que el pasaje de A 20/B 34 no parece calzar del todo con la lectura del árbol del pasaje clasificatorio, lo cual parece debilitarla.

Por razones de espacio, no puedo aquí reconstruir con precisión y detalle cómo debería ser interpretado el pasaje clasificatorio. Con todo, me parece claro que más que clases, géneros y especies de cosas, Kant, tanto en A 20/B 34 como en A 320/B 376-7, está más bien distinguiendo entre momentos de una única realidad y no entre entidades diferentes como parece sugerir la lectura del árbol. No hay en A 320/B 376-7, de este modo, una clasificación de las representaciones identificando géneros y especies, sino más bien la identificación de diferentes momentos de la experiencia que tenemos del mundo, momentos que pueden ser identificados por referencia a diversos aspectos de la representación, según sea considerada desde diferentes puntos de vista (como dependiente de la sensibilidad, como derivando su contenido de la experiencia o no, etcétera). Es de destacar, de hecho, que un árbol de Porfirio distingue clases de entes, mientras que no toda clasificación con forma de árbol debería entenderse inmediatamente como una clasificación de entes en clases. De este modo, el carácter clasificatorio 
del pasaje puede eventualmente mantenerse, pero sin considerar que los relata guardan entre sí el tipo de relaciones que guardan los elementos de un árbol de Porfirio.

Más allá de lo anterior, lo que parece ser aún más claro es el hecho de que la aparente claridad en la definición que Kant proveería aquí sobre la intuición desaparece completamente cuando se toma en cuenta i) que no es para nada claro en qué sentido ha de entenderse que la intuición es conocimiento singular e inmediato (Grüne 46 y ss.; Smit 235) y ii) que no es claro si las aparentes notas definitorias del concepto de intuición son realmente tales (lo anterior es una consecuencia clara de la puesta en cuestión de la lectura del árbol del pasaje clasificatorio, aunque puede defenderse a partir de otros argumentos). En lo que sigue realizaré un breve análisis de estos puntos. El objetivo del análisis no será dirimir por completo la cuestión del significado de las notas de singularidad e inmediatez de la intuición, asunto que ha sido ampliamente discutido en la literatura reciente, sino contribuir a mostrar con mayor claridad hasta qué punto la lectura que sostiene la prioridad lógica de la noción de la intuición sobre la intuición sensible e intelectual ha de ser puesta en cuestión. Para ello intentaré dar cuenta brevemente de cómo, a la luz de las discusiones más recientes, parece ser razonable interpretar estas condiciones de la intuición en tanto habrían de identificar un concepto o bien unívoco, o bien principal, pleno o focal de intuición.

\subsection{Sobre el significado de los términos inmediato y singular (i)}

De acuerdo con la pretendida definición kantiana de intuición que veíamos en pasajes como A 320/B 376-7 o AA 20 266, 14-8, la misma sería un tipo de representación inmediata y singular. Ahora bien, no queda del todo claro qué es aquello que se mienta por estos términos que son, creo, susceptibles de múltiples interpretaciones, tal como se puede apreciar en la ingente porción de la literatura secundaria que se ha dedicado a aclarar el significado de estas nociones. Partamos con el caso de la singularidad: 
una primera manera de entender el asunto es pensar que una intuición es un tipo de representación que se refiere a un y solo un objeto, mientras que un concepto se referiría a una pluralidad de objetos. Entendida de esta manera, la intuición jugaría un papel idéntico o al menos similar al que juegan en la semántica estándar los nombres propios, esto es, expresiones que refieren a un y solo un objeto. Con todo, es claro que no siempre los conceptos representan una multiplicidad de objetos. El concepto luna de la tierra refiere a un y solo un objeto, así como el concepto Copas Libertadores ganadas por la Universidad de Chile refiere a ninguno. Ahora bien, sabemos que en rigor todo esto es contingente (Grüne 46), pues bien podría ocurrir, al menos desde el punto de vista de aquello que es lógicamente concebible, que en el futuro el concepto luna de la tierra sea satisfecho por un conjunto de individuos mayor a uno. Nada hay, en efecto, de contradictorio en la idea de que la tierra tenga dos lunas. Esto quiere decir que la diferencia entre intuiciones y conceptos no puede ser comprendida como una tal que se funde en el hecho de que la intuición está de facto por un y solo un objeto, mientras que los conceptos están de facto por varios objetos. La diferencia podría ser considerada como más bien sosteniendo que las intuiciones no pueden referir a más de un objeto, mientras que los conceptos, sí (como podría ocurrir que luna de la tierra designara no ya a una sola cosa, sino que a dos, entonces tiene sentido llamar a luna de la tierra un concepto). Grüne ha sugerido de manera sumamente interesante que, de todos modos, el asunto podría ser más complicado, puesto que cabe imaginar la posibilidad, según el modo en que Kant usa el término, de una intuición que comprenda una cantidad mayor a uno de objetos, por ejemplo, una intuición de diez árboles. Para abordar este punto Grüne sugiere un criterio interesante: una intuición se diferenciaría de un concepto ante todo por el hecho de que ella representa lo que de facto representa. Por el contrario, un concepto sería general toda vez que representa más que aquellos objetos que de facto representa. Así, por ejemplo, el color rojo comprende bajo sí a las múltiples representaciones que lo tienen como nota común, siendo estas representaciones infinitas o potencialmente infinitas (B 39-40). La intuición que tengo de estas manzanas rojas, en cambio, solo representan a estas 
manzanas rojas. Se podría decir, quizás, que la misma idea es expresable de la siguiente manera: una intuición es un tipo de representación que guarda una correspondencia biunívoca con lo representado, esto es, existe una relación uno a uno entre lo representado y la representación. No ocurriría lo mismo con los conceptos, toda vez que ellos representan mucho más que el mero concepto, es decir, la extensión posible del mismo.

En el caso de la distinción mediato/inmediato, parece ser más fácil de comprender la diferencia que habría entre intuiciones y conceptos, pero solo en apariencia. De todos modos baste por ahora decir que se trata fundamentalmente de una distinción que parece remitir al modo en que las representaciones refieren a objetos, y no tanto a qué tipo de objetos son referidos. La idea parece ser que solo hay una referencia inmediata a los objetos, ahí donde ellos no son referidos por medio de notas que son comunes a más cosas o que no se refiere por medio de otra representación al objeto. El concepto rojo, por ejemplo, referiría a las cosas rojas a través de las notas que ellas comparten por ser rojas. No ocurriría lo mismo con la intuición que tenemos de un objeto singular. Por ello en la intuición tendríamos conocimiento directo. Por otra parte, en el caso del juicio 'los cuerpos son pesados', el predicado pesado se referiría a los objetos a los que les corresponde a través del sujeto cuerpos (A 68/B 93 y A 69/B 94). Entendidas las notas de intuición de esta manera podríamos eventualmente haber ganado un concepto unívoco, pleno o focal de intuición. No obstante, como vimos más arriba en el punto (ii), hay más complicaciones que atender. Las tendré en cuenta en la medida que su consideración permite dar cuenta del objetivo de este texto.

2.2. Sobre las notas definitorias del concepto de intuición (ii)

Para muchos autores no es completamente transparente que Kant haya entendido la singularidad y la inmediatez como condiciones necesarias definitorias de la intuición. Es menester hacer notar que existen autores que sostienen, basados en algunos de los textos kantianos referentes al término 
intuición, que existe solo un elemento que es central en su definición y el segundo un agregado más bien accidental, o si se quiere lógicamente posterior. Esta línea de interpretación tiene su origen en un sugerente artículo de Hintikka (1992), aunque en rigor la inspiración de Hintikka remonta a trabajos anteriores de E. Beth, y en trabajos posteriores que generaron una recorrida línea de interpretación de la filosofía de las matemáticas de Kant. En este texto, que se ha transformado en un pequeño clásico de la interpretación kantiana en el ámbito de la filosofía de las matemáticas, Hintikka sostiene que el carácter fundamental de la intuición kantiana es ser un conocimiento singular: "Cualquier cosa que se representa la mente humana como un singular (individual) es una intuición. No existe nada 'intuitivo' en la intuición así definida. La intuitividad significa simplemente singularidad" $(23)^{9}$. Hintikka intenta sostener su interpretación en varios puntos. Uno de ellos sería el hecho de que ella mostraría que el desarrollo de métodos formales de prueba no haría obsoleta la concepción kantiana de la demostración matemática así como el papel que en ella desempeña la noción de construcción. Para que lo anterior quede claro, Hintikka intenta dar cuenta del modo en que habitualmente sería comprendido el aporte de la intuición en la construcción matemática. Este aporte correspondería, en esta visión tradicional, a una suerte de visualización que permitiría a los matemáticos realizar demostraciones apelando a procedimientos no estrictamente lógicos (esta manera de comprender el aporte de la intuición en Kant se halla, por ejemplo, en un clásico texto de Russell que el mismo Hintikka cita). Ahora bien, esta manera de entender el aporte de la intuición en la matemática como apelando a procedimientos no estrictamente lógicos

9 Esta cuestión ha sido muy debatida en la literatura sobre la noción de intuición desde los trabajos de Hintikka. Mientras algunos autores contemporáneos simpatizan con Hintikka en lo relativo a la prioridad de la singularidad (Simian 130-1), hay quienes han defendido una cierta identidad entre ambos (Wilson) y quienes han sostenido más bien la prioridad de la nota de inmediatez (Torretti 116, que sigue en esto el trabajo de Parsons) Desde el punto de vista textual, ambos modelos parecen tener cierto respaldo (para la interpretación de Hintikka, cfr. A 32/B 47; AA 09 91; AA 24 451; AA 24 566; AA 24 653; AA 24 904-905; R 2836; para la interpretación de Parsons y Torretti, AA 04 281). 
sería deudora del modo en que Kant concibe la intuición en la "Estética trascendental”, pero no en el modo en que comprende este concepto en toda su obra. De acuerdo con Hintikka, la concepción de la intuición de la "Estética trascendental" sería tanto sistemática como históricamente posterior a aquella que concibe la nota de singularidad como el elemento definicional de la intuición. Entre otras cosas, destaca Hintikka, lo anterior se sustentaría en que la conexión entre sensibilidad e intuición no podría estar implicada en los elementos definitorios de la noción misma de intuición, toda vez que Kant insiste a lo largo de la $K r V$ en que "no es incomprensible que otros seres puedan tener intuiciones por medios diferentes a los sentidos" (23).

Hintikka, junto con lo anterior, menciona una importante cantidad de pasajes de Kant que respaldarían su lectura, aunque en rigor no todos calzan con lo que él quiere sostener ${ }^{10}$. De los textos que Hintikka menciona, el más conocido y favorable a su interpretación es el que toma a partir de una observación de Frege, que corresponde al $\$ 1$ de las "Lecciones de lógica” según la versión de G. B. Jäsche, donde Kant señala:

Todos los conocimientos, es decir, todas las representaciones referidas con conciencia a un objeto son o bien intuiciones o conceptos. La intuición es una representación singular (raepresentatio singularis), el concepto una general (raepresentatio per notas comunes), o representación reflexiva (raepresentatio discursiva). (AA 0991 6-10)

Luego de realizar esta operación de reducción, por así decir, de la interpretación tradicional, Hintikka sostiene que se puede comprender mejor el papel que la intuición en Kant juega en la matemática, y en particular en la geometría, e incluso expresar ese papel de modo riguroso y comprensible apelando a métodos formales. Para ello, sería necesario no perder de vista los modelos que Kant tiene en mente a la hora de proponer su concepción de la geometría. Como es sabido, este modelo es ante todo el de Euclides. De acuerdo con la clásica sistematización de la demostración en

10 $\mathrm{Al}$ respecto véase Placencia 75, n 121. 
los Elementos realizada por Proclo en su comentario a esta obra (Comentario 203), toda demostración posee al menos cinco partes. En primer lugar está la enunciación o prótasis (por ejemplo en la proposición veinte "En un triángulo dos de los ángulos tomados juntos son siempre mayores que el tercero"). Luego, Euclides siempre, según Hintikka, aplica el contenido de la enunciación a una figura que se asume dibujada. Este es el procedimiento que Proclo llamó ékthesis. En el caso de la proposición veinte se trataría, por ejemplo, del siguiente paso "Sea $\mathrm{ABC}$ un triángulo. Digo que en $\mathrm{ABC}$ dos lados tomados juntos son mayores al tercero. $\mathrm{AB}$ y $\mathrm{AC}$ son mayores que BC... etc.”. En tercer lugar, Euclides dispone de construcciones auxiliares (kataskeué), paso en el que en ocasiones la figura presentada en la ékthesis es complementada con otras figuras que ayudarán en el cuarto paso, la demostración o apódeixis, a lo que sigue la enunciación general donde se pasa de la conclusión de la figura presentada en la ékthesis a una formulación general de la misma (Hintikka 28-9). Hintikka sostiene que de acuerdo a Kant la intuición está a la base de la ékthesis y de la kataskeué, lo que Kant expresaría de otro modo indicando que la intuición es el tipo de representación que está a la base de la construcción, operación que distinguiría al conocimiento matemático de aquel que procede por análisis de conceptos (30). Lo anterior permitiría, por último, presentar un análisis de la concepción kantiana de la matemática que sería expresable además en términos de la regla de inferencia conocida en los sistemas de deducción natural de la lógica moderna como ejemplificación existencial, regla que expresaría de modo preciso lo que la ékthesis quiere expresar (35). Esta regla permite deducir de una proposición de la forma $\exists x F(x)$ una proposición del tipo $F(u)$ en que se reemplaza la variable ligada por una constante. Se trata, como ha destacado Torretti (122), de un modo de proceder habitual en geometría: supuesta la existencia de objetos de una cierta condición, se propone considerar a uno de ellos cualquiera para el que se muestra la posesión de una determinada propiedad.

Con todo, todos estos argumentos parecen tener problemas relevantes. Por de pronto, no parece ser del todo claro que Kant quiera tener la intuición operando solo en aquellos casos que son reconducibles a la ékthesis. Kant 
sostiene que la construcción no solo opera en la demostración (entendida aquí en sentido amplio, no solo como la parte de la demostración que Proclo llama apódeixis), sino que también en las definiciones y axiomas (A 730/B 758; A 732/B 760). Nada de esto parece poder ser asumido por la posición desarrollada por Hintikka. Tampoco parece ser claro que en un intento de expresar de manera detallada y precisa la estructura completa de una demostración empleemos una ejemplificación existencial ahí donde corresponde usar la ékthesis. De hecho, ejercicios realizados por E. Beth en trabajos cruciales para Hintikka muestran que para, por ejemplo, el caso de la proposición 32 del libro primero de los Elementos de Euclides (la suma de los ángulos interiores de un triángulo es igual a dos rectos), no es necesario realizar ninguna ejemplificación existencial (Beth 373). Se podría pensar que lo correcto sería entonces trasladar la ejemplificación existencial a la enunciación, pero eso generaría un problema, pues no pareciera ser lícito pasar del cuantificador existencial contenido en la ejemplificación existencial al cuantificador universal que se emplea en la enunciación general, al ser universalizado el resultado de la prueba desarrollada por el geómetra para una figura arbitraria ${ }^{11}$.

No obstante, no es ese el punto que me interesa más aquí, sino más bien el siguiente: uno de los principales argumentos de Hintikka para sostener que la noción de intuición no tiene nada que ver con la sensibilidad es el hecho de que puede haber otras formas de intuición; lo cual mostraría, además, que la concepción que pareciera presentar Kant en la "Estética trascendental" sería derivada de una concepción de la intuición que admitiría ser comprendida como desconectada de la sensibilidad y, por tanto, de la nota de inmediatez y, en consecuencia, que, según parece asumir Hintikka, solo sería necesaria al asumir que la intuición está ligada a la sensibilidad. Esta manera de entender las cosas parece suponer que el concepto de intuición ha de ser aclarado en términos genéricos para luego ser entendido en el modo en que Kant lo caracteriza al ser determinado como intuición humana y como intuición intelectual. Hintikka claramente

11 Para diversos puntos críticos de la posición de Hintikka, véase Fuentes (264 y ss). 
cree hacer esto al caracterizar la noción de intuición como conocimiento singular. Dicho de otro modo, la posición de Hintikka parece suponer la anterioridad -al menos lógica- del concepto de intuición en relación con el de intuición sensible, al modo en que un género es anterior, al menos lógicamente, a sus especies. Así las cosas, tanto Hintikka como muchos otros intérpretes de esta noción en la obra de Kant, operan teniendo en cuenta un modelo que podríamos llamar porfiriano, esto es, un modelo en el que se toma la noción de intuición como un concepto general que tendría especies, a saber, la intuición humana (en tanto intuición de un ser finito) y la intuición intelectual (en tanto intuición de un ser infinito), junto con otras eventuales especies de intuición sensible que podrían responder a formas diferentes de la sensibilidad. Para que esto pueda funcionar como modelo del modo en que Kant entiende la relación entre las nociones de intuición, intuición sensible e intuición intelectual, es necesario que las especies tengan en común al género y que compartan las características que satisface el género ${ }^{12}$.

Así las cosas parece ser que el intento de Hintikka por entender el aporte de la noción de intuición en las matemáticas nos lleva a una comprensión de la noción que resulta interesante ya no solo para quienquiera que se interese por el problema de la filosofía de las matemáticas en Kant, sino también para quien se interese por la noción de intuición intelectual, que es empleada aquí como un recurso para mostrar que la referencia a la sensibilidad y con ello a la inmediatez en la caracterización de la noción de intuición, es innecesaria, así como para quienquiera que se interese por la noción de intuición en general. En lo que viene, mi objetivo será revisar si el concepto de intuición intelectual puede ser entendido como una especificación del concepto de intuición, tal como parecen entender Grüne, Hintikka o Kern este género, esto es, como una representación singular o bien inmediata y singular, entendidas además estas características tal como fueron presentadas anteriormente en el punto (i).

12 La idea básica de Hintikka aquí, a saber, el esquema porfiriano, es compartida por autores diversos, como Grüne (49) o Kern (110-2). 


\section{INTUICIÓN INTELECTUAL}

Todo esto parece sugerir que la noción misma de intuición intelectual tiene un contenido claro. No obstante, al igual que la noción de intuición, la de intuición intelectual parece ser oscura en $\mathrm{Kant}^{13}$. Junto con ello se trata de un tópico comparativamente poco tratado por la literatura kantiana. En primera instancia, debe destacarse que, al igual que ocurre con la noción misma de intuición, no es del todo claro si ella, de hecho, tiene solo un significado o más. Aunque algunos especialistas (v. gr. Kern 210-2) parecen entender que el concepto es unívoco o al menos no llaman la atención sobre la posible equivocidad del mismo, otros autores han distinguido, con razón a mi modo de ver, al menos dos sentidos de este término en este autor (Gram 287-304; Förster Die 25 Jahre der Philosophie 159). Así por ejemplo Gram (288) afirma que Kant emplea un solo término, a saber, intuición intelectual, para designar tres asuntos distintos: i) (el acto de) un entendimiento que conoce las cosas en sí mismas, ii) (el acto de) un entendimiento que intuiría la totalidad de los fenómenos, y iii) (el acto de) un entendimiento que crearía su propio objeto. Lo anterior, al menos a primera vista, calza bien con los textos. En efecto, por un lado, Kant parece, en ocasiones, designar por medio de intuición intelectual o de un entendimiento que intuiría a la capacidad de un entendimiento arquetípico que intuiría sus objetos sin necesidad de que ellos les sean dados de modo tal que incluso él los produciría por medio del pensar (B 71-72; B 145). Tal concepción de un entendimiento arquetípico, que aparece ya en la Dissertatio, es mencionada por Kant también en la famosa carta a Herz de febrero de 1772:

Si aquello que se llama en nosotros representación fuera activa en consideración del objeto, es decir, si incluso el objeto fuera creado por medio de ella, tal como uno se representa el conocimiento divino siendo el prototipo de las cosas, entonces podría pensarse la conformidad de las mismas con el objeto. (AA 10130 17-21)

$13 \quad \mathrm{Al}$ respecto, véanse Giannetto (17 y ss.) y Tilliette (13-36) 
Otro tanto ocurre en un importante pasaje de la "Estética trascendental", donde Kant compara nuestro intuir con un intuir originario:

No queda nada de ellas, si no se las quiere hacer formas objetivas de todas las cosas, que el hacerlas formas subjetivas de nuestro modo de intuir externo así como interno que por ello se llama sensible, porque no es originario, esto es, no es una tal por él que se nos dé la misma existencia del objeto de la intuición (y que, de acuerdo a lo que comprendemos, solo le puede corresponder al ser originario), sino que es dependiente de la existencia del objeto, por lo tanto solo es posible por el ser afectada por el objeto la capacidad representativa del sujeto. (B 71-72)

Esta idea parece ser muy relevante en el marco de pasajes tan cruciales como la "Deducción trascendental de las categorías", donde Kant destaca que su argumentación en la primera sección de la misma ( $\$ \$ 15-21)$, no obstante no hacer ninguna suposición respecto del modo en que se le da lo múltiple a ser enlazado, no se ha podido abstraer del hecho de que eso múltiple debe ser dado:

Sin embargo, no pude dejar de lado un punto en lo anterior: que lo múltiple para la intuición debe ser dado para la síntesis del entendimiento antes de ella y de modo independiente de ella. Cómo tenga lugar esto, queda indeterminado. Puesto que si hubiera querido pensar un entendimiento que intuyera él mismo (como es el caso del entendimiento divino que no se representa objetos dados, sino que se los da o los produce por medio de su representación), entonces las categorías no habrían tenido ninguna significación en relación con este conocimiento. (B 145)

Hasta acá, parece haber un significado claro del término intuición intelectual o de expresiones semejantes (el acto cognitivo de un intelecto arquetípico, etcétera). Se trataría de expresiones que designarían un tipo de intuición que no requeriría que el objeto le sea dado, sino que sería tal que ella misma crearía al objeto. Pero las cosas son más complicadas, porque 
Kant parece emplear el término de otros modos. Así, por ejemplo, en otros casos el término intuición intelectual es empleado para referirse al modo en que intuiría un entendimiento de carácter no sensible, y que por tanto presentaría el objeto como no mediado por nuestras condiciones subjetivas de representación (B 307), esto es, como el acto de un entendimiento que captaría el noumeno en sentido positivo. Así, por ejemplo, ocurre en el siguiente pasaje: "Si entendemos por noumeno el objeto de una intuición no sensible, entonces aceptamos un modo de intuición particular, a saber, la intuición intelectual, que no es la nuestra, y cuya posibilidad no podemos comprender" (B 307) ${ }^{14}$. Como bien destaca Gram, esta concepción de la intuición intelectual apunta a un aspecto distinto que la primera antes mencionada. En el segundo caso se trata de la posibilidad de usar conceptos puros para conocer noumena en sentido positivo o cosas en sí mismas, con independencia de las condiciones subjetivas que permiten que los seres humanos tengamos experiencia de fenómenos; mientras que en el primer caso se trata de la posibilidad de que un entendimiento cree los objetos con su mero pensar.

Con todo, esto no es el único caso en que Kant emplea expresiones del tipo de intuición intelectual. Es conocido que en los $\$ \$ 76-7$ de la Crítica de la facultad de juzgar Kant introduce la noción de un intelecto intuitivo:

Nuestro entendimiento tiene por tanto la propiedad de que debe ir en su conocimiento, por ejemplo, de la causa de un producto, de lo analítico-universal (de los conceptos) a lo particular (la intuición empírica dada) [...] Ahora, nosotros podemos pensar también un entendimiento que, por ser intuitivo y no discursivo como el nuestro, va de lo sintético-universal (de la intuición de un todo como tal) a lo particular. (AA 05 407)

Como bien destaca Gram (293), esta noción de intuición intelectual se ha apartado de sus dos precedentes. Se trata, acá, de una noción de

$14 \quad$ Es de notar que Kern (212) presenta todos estos pasajes como si se tratara del mismo uso del término. Esto me parece un error, puesto que si bien es cierto es pensable que ambas formas designen el modo de conocer de una misma entidad, no es de suyo evidente que así deba ser. 
intuición que correspondería a la capacidad de intuir de una vez la totalidad de los fenómenos que constituyen nuestro mundo. Gram agrega, que esta idea "no implica la pretensión de que un objeto dado independiente de las condiciones de la sensibilidad, ni implica la pretensión de que un entendimiento puede crear el objeto que conoce por pensarlo" (293) ${ }^{15}$.

Por cierto, siempre existe la posibilidad de que estas maneras de caracterizar la noción de intuición intelectual, respondan en rigor al mismo objeto. Con todo, no parece a primera vista ser claro que eso sea lo que Kant tiene en mente. Más interesante para mis propósitos, me parece el hecho de que Kant parece caracterizar en algunos pasajes la noción de intuición entendida como nuestra intuición de un modo negativo frente a la noción de intuición intelectual sin aludir en ningún caso a un núcleo común de ambas. Dicho de otro modo, Kant parece con esto al menos no dar evidencia para el punto de Hintikka, en la medida en que no sostiene que haya algo así como un núcleo común de la intuición dado por la singularidad que sea propio tanto del conocimiento de la intuición intelectual como de la intuición humana. Cabe entonces aquí hacer la pregunta: ¿Qué hace de una intuición sensible y de una intuición intelectual una intuición? ¿ Son ellas dos especies de un mismo género, o bien el término intuición está aplicado aquí de modo equívoco? Más aún, es digna de hacerse la cuestión sobre si estas preguntas se pueden responder habida cuenta el hecho de que Kant mismo sostiene de la intuición intelectual que es un tipo de conocimiento "cuya posibilidad no podemos comprender" (B 307).

\section{INTUICIÓN SENSIBLE E INTUICIÓN INTELECTUAL}

Sabemos que la intuición es, en la caracterización tradicional, un tipo de conocimiento singular e inmediato. Curiosamente, ninguna de estas

\footnotetext{
15 Junto con lo anterior recientemente en la investigación se ha intentado distinguir la noción de intuición intelectual de la noción entendimiento intuitivo (Förster "Die Bedeutung” y Die 25 Jahre 158 y ss.), que dejaré aquí, por razones de espacio, fuera de consideración.
} 
características es afirmada o negada de la intuición intelectual en ninguno de los pasajes antedichos ni en los pasajes que conozco en que Kant emplea esa expresión, por lo que no parece haber evidencia, al menos a primera vista, para suponer que aquella sea una suerte de género a partir del cual se individualicen formas específicas de intuición ${ }^{16}$. Obviamente esto excluye el hecho de que la frase intuición intelectual se compone del sustantivo intuición, el cual, no obstante, como ya hemos visto más arriba, ni siquiera es, con una alta probabilidad, empleado por Kant de modo unívoco. Al tratarse de la intuición intelectual, por su parte, solo se nos dice que ella corresponde a un tipo de intuición que no requiere que el objeto le sea dado o cuyo objeto no es sensible sino intelectual o bien se podría pensar que se nos dice que ella es el fruto de un entendimiento intuitivo tal como es caracterizado en la Kritik der Urtheilskraft $(K U)$. Todo lo anterior se vuelve tanto o más complicado cuando constatamos que Kant observa respecto de Dios, que suele ser considerado como aquella entidad de la que podríamos concebir que ella poseyera una intuición intelectual, que no podemos determinar de modo concluyente cuáles son sus características, lo que incluye por cierto el modo en que él conocería. En efecto, solo podemos determinar por vía negativa y de eminencia a partir del modo en que nosotros conocemos ${ }^{17}$. Me parece que esto queda claro, por ejemplo, a propósito del siguiente pasaje:

El concepto de un noumeno, tomado de modo meramente problemático, permanece siendo, no obstante, no solo permisible, sino que imprescindible como un concepto que le pone límites a la sensibilidad. Pero entonces él no es un objeto inteligible particular para nuestro entendimiento, sino que un

16 El único esfuerzo que conozco por intentar mostrar hasta qué punto la intuición intelectual sería compatible en lo relativo a sus notas características con una especificación del término intuición es el texto de Giannetto (9-32). Con todo, me parece que este trabajo presenta diversos problemas (por de pronto no se hace cargo de la discusión contemporánea sobre la diversidad de modos en que puede ser entendidas las pretendidas notas definitorias).

17 Un buen análisis de cómo esto se realiza ha sido llevado a cabo recientemente por L. Pelegrín. 
entendimiento al que perteneciera este objeto sería él mismo un problema, a saber el de conocer su objeto no discursivamente por medio de categorías, sino intuitivamente en una intuición no sensible, entendimiento de cuya posibilidad no nos podemos hacer la menor representación. (A 256/B 311-2) ${ }^{18}$

De aquí, me parece, se sigue que el estudio del concepto de intuición intelectual no solo se halla con la dificultad de que Kant emplea este concepto y afines con múltiples significados, sino que también el hecho de que Kant mismo parece ser escéptico respecto de la posibilidad de que nos podamos representar de modo cierto cuál sería el modo preciso de conocimiento de un entendimiento que intuyera (Willaschek 336). Así las cosas, mientras nos podemos hacer una idea relativamente precisa de la intuición humana, no parece ser que nos podamos hacer una idea igualmente precisa de otro tipo de intuición. Esto parece atentar contra la idea de que el concepto de intuición intelectual es una especie de un género común al que pertenecería la intuición humana, sino que más bien lo presenta como una suerte de concepto cuya posibilidad no es del todo decidible y, por tanto, cuyas condiciones de pertenencia a un género no parecen poder ser aclaradas plenamente. Ante esto, la mejor estrategia para elucidar el contenido de la noción de intuición intelectual y revisar si existe alguna noción de intuición que preceda a la de intuición sensible e intuición intelectual es volver al concepto de intuición humana. Volvamos a la caracterización de intuición humana o intuición sensible y a la caracterización positiva de la misma:

Sea cual sea el modo y los medios por el que un conocimiento se refiere a objetos, la intuición es aquel a través del cual ellos se refieren de modo inmediato a objetos y al que todo pensamiento se dirige como medio. La intuición solo tiene lugar en tanto el objeto nos es dado. Esto solo es posible, al menos para nosotros los seres humanos, en la medida en que el objeto afecta de cierta forma al ánimo. La capacidad de recibir representaciones por medio del modo

18 La expresión entendimiento es una de las posibles lecturas del texto. Sigo aquí el modo en cómo entienden el texto autores como M. Caimi en su traducción castellana o M. Willaschek. 
en que somos afectados por los objetos, se llama sensibilidad. Por medio de la sensibilidad nos son dados los objetos, y solo ella nos trae intuiciones. Por medio del entendimiento los objetos son pensados, y de él surgen conceptos. Por lo tanto, todo pensamiento, en nuestro caso, debe dirigirse a las intuiciones, ya sea de modo directo o indirecto, por medio de ciertas notas, porque el objeto no nos puede ser dado de otro modo. (A 19/B 33)

Este pasaje, creo, deja claro que la intuición humana se caracteriza por depender de que el objeto nos sea dado. Esta es una representación que nos presenta los objetos de un modo específico: directo o si se quiere, inmediato. Interpretamos más arriba inmediato como aludiendo al hecho de que la intuición refiere al objeto sin referir a su vez a otras representaciones, notas, conceptos o intuiciones. Ahora bien, como se ha dicho, la tesis del texto citado es que este modo de representar inmediato solo puede presentarnos aquellos objetos que nos son dados. No tenemos conocimiento directo de nada que no nos afecte, porque nuestro conocimiento no crea los objetos. Como se ve aquí, la idea, problemática, de intuición intelectual, al menos en el sentido (iii) del término que fue distinguido más arriba, sirve meramente de contraste para que quede más claro cuál sería el carácter de la intuición tal como la está caracterizando Kant aquí.

Así las cosas, a falta de un pasaje en el que Kant define el concepto de intuición elucidando explícitamente si tal concepto es definido con independencia del modo humano de intuir y no quedando del todo claro cuáles son las notas de su concepto de intuición intelectual (concepto que además, se nos dice en ocasiones, puede ser incluso problemático), solo cabe inferir que no parece ser del todo claro que sea legítimo concluir a partir de las afirmaciones de Kant en la $\mathrm{KrV}$ que el concepto de intuición humana está pensado como especie de un género mayor caracterizado por ser conocimiento singular. Más bien parecen haber razones para lo contrario. En efecto, en mi opinión, una de las condiciones bajo las cuales parece tener sentido afirmar el carácter singular e inmediato de la intuición sensible es justamente la de que un objeto sea dado. Pero es precisamente esta condición la que no se verifica, al menos en el sentido (iii) de intuición 
intelectual. La intuición intelectual en la medida en que crea su objeto podría crear su objeto sin representar eo ipso solo aquello que corresponde de facto uno a uno con el objeto creado. Tampoco eso creado es conocido necesariamente de modo inmediato, esto es, como representando su objeto sin mediación de otras representaciones. Al ser todas estas posibilidades al menos concebibles, no descartadas por Kant y a la vez compatibles con la idea de que la intuición intelectual crearía su objeto en vez de tener que serle este dado, no parece ser razonable pensar la idea de un intuición intelectual como una especie de un género común llamado intuición.

Por lo demás, cabe destacar que la intuición intelectual parece responder más bien, al menos en el sentido (iii), al modelo de lo que Kant llama conocimiento práctico: un tipo de relación en la que la representación realiza al objeto y no el objeto se rige por la representación (B IX-X). Pero ocurre que ese tipo de conocimiento es heterogéneo respecto del conocimiento teórico, que es el que Kant trata siempre que se trata de hablar de la noción de intuición. La intuición sensible, de hecho, no puede ser conocimiento práctico en la medida en que ella solo es posible a partir de la donación del objeto.

Así las cosas, la intuición intelectual parece ser, más bien, una suerte de determinación de un tipo de conocimiento completamente distinto en género al nuestro, pero que solo podemos conocer por vía negativa, de eminencia y por analogía a partir de nuestro conocimiento (AA 28 1021 y ss. ${ }^{19}$. Tomamos así las capacidades cognitivas humanas y negamos características de ellas como por ejemplo conocer solo aquello que nos es dado, o bien conocer bajo las condiciones de nuestra subjetividad, o bien conocer de modo mediato y sin que nos pueda ser dada de una vez la totalidad de la experiencia, y ahí tenemos entonces como resultado los sentidos ya mencionados de la intuición intelectual. Este es el modo en el que, de hecho, Kant asigna predicados a Dios en las Lecciones de religión (AA 28 1021).

19 Cómo ocurre esto es tratado con claridad por Pelegrín (43-59). 


\section{Conclusión}

Si es correcto lo que se ha indicado aquí, entonces no parece ser razonable asumir la tesis de que la intuición es una suerte de género común de la intuición sensible y la intuición intelectual. Con ello pareciera quedar descartada, aunque no toda posibilidad de que el concepto de intuición sea lógicamente anterior al de intuición intelectual (en efecto, la anteroposterioridad lógica no es reductible sin más a las relaciones entre género y especie en la variante del árbol de Porfirio), sí al menos la manera más tradicional de sostener la tesis de la prioridad lógica. De este modo, la tesis de que la intuición es lógicamente anterior a las presuntas especies sensible e intelectual parece perder buena parte de su sustento y quien quisiera seguir sosteniéndola debería hacerse cargo de explicitar de qué modo -que no sea el de la relación de género y especie- podría ser concebida esta prioridad. A la luz de lo visto parece, por el contrario, más auspicioso asumir que el concepto de intuición intelectual es en un sentido relevante completamente heterogéneo respecto del de intuición sensible y que este último es anterior o primero no solo quoad nos sino que también en sentido lógico, en la medida en que el concepto intuición intelectual solo es definible o caracterizable por recurso a la intuición sensible o a las capacidades cognitivas humanas. Con todo, al ser el entendimiento intuitivo - de haberlo- el fundamento de todo otro entendimiento, parece ocurrir que sí tiene sentido afirmar que este entendimiento así como su acto es ontológicamente anterior al entendimiento y la sensibilidad humanas, así como a sus actos. Pero parece ser que al menos en el marco de la filosofía kantiana no nos está dado poder comprender lo anterior de ese modo. 


\section{Bibliografía}

Aristóteles. Aristotle's Metaphysics. Introducción y comentarios de W. D. Ross. Oxford: Clarendon, 1924.

Allison, Henry. Kant's Trascendental Idealism. An Interpretation and Defense. New Haven-Londres: Yale UP, 2004.

Beth, Evert. "Über Lockes allgemeines Dreieck". Kant-Studien, n.o 48, 1956-1957, pp. 361-80.

Ewing, A. C. A short Commentary on Kant's Critique of Pure Reason. Chicago: University of Chicago Press, 1938.

Falkenstein, Lorne. Kant's Intuitionism. A Commentary on the Transcendental Aesthetic. Toronto: University of Toronto Press, 1995.

Förster, Eckart. "Die Bedeutung von $\$ \$ 76-77$ von Kants Kritik der Urteilskraft für die Entwicklung der nachkantischen Philosophie". Zeitschrift für Philosophische Forschung, vol. 56, n. ${ }^{\circ}$ 2, 2002, pp. 321-45.

_. Die 25 Jahre der Philosophie. Fráncfort del Meno: Klostermann, 2012.

Fichte, Johann. J. G. Fichte Gesamtausgabe. Editado por R. Lauth und H. Jacob. Stuttgart-Bad Cannstatt: Frommann Verlag, 1964.

Friebe, Cord. "Anschauung". Kant-Lexikon. Editado por Willaschek, Stolzenberg, Mohr y Bacin. Berlín-Boston: Walter de Gruyter, 2015, pp. 100-3.

Fuentes, Javier. "Conexiones entre Kant, Proclo y Euclides a partir de una interpretación de Hintikka”. Con-Textos Kantianos, n. ${ }^{\circ}$ 5, 2017, pp. 261-77.

Giannetto, Giuseppe. Intuizione intellettuale e sintesi trascendentale in Kant. Nápoles: La scuola di Pitagora editrice, 2014.

Gram, Moltke. "Intellectual Intuition: The Continuity Thesis". Journal of the History of Ideas, vol. 42, n. ${ }^{\circ}$ 2, 1981, pp. 287-304.

Grüne, Stefanie. Blinde Anschauung. Fráncfort del Meno: Klostermann, 2009.

Hintikka, Jaakko. "Kant on the Mathematical Method". Kant's Philosophy of Mathematics. Editado por Carl Posy. Dordrecht: Kluwer, 1992, pp. 1-42.

Kant, Immanuel. Kant's gesammelte Schriften [AA]. Edición de la Academia de Ciencias de Prusia . Berlín: 1902. 
_. Kritik der reinen Vernunft $[\mathrm{Kr} V]$. Editado por Raymund Schmidt. Hamburgo: Felix Meiner, 1956.

_. Crítica de la razón pura. Traducido por M. Caimi. Buenos Aires: Colihue, 2018.

Kern, Andrea. "Anschauung, intellektuelle". Kant-Lexikon. Editado por Willaschek, Stolzenberg, Mohr y Bacin. Berlín-Boston: Walter de Gruyter, 2015, pp. 110-2.

Lazos, Efraín. Disonancias de la crítica, México D. F.: UNAM, 2014.

McDowell, John. Mind and World. Harvard: HUP, 1996.

Parsons, Charles. "Kant's Philosophy of Arithmetic". Kant's Philosophy of Mathematics. Editado por Carl Posy. Dordrecht: Kluwer, 1992, pp. 43-79.

Paton, H. J. Kant's Metaphysic of Experience. Londres: George Allen \& Unwin, 1936.

Pelegrín, Laura. "Dios conoce todo a priori, tiene un intelecto puro e intuitivo. La determinación kantiana de los predicados psicológicos de Dios por vía especulativa”. Ideas y Valores, vol. 65, n. ${ }^{\circ} 161,2016$, pp. 43-59.

Placencia, Luis. La ontología del espacio en Kant. Un estudio genético-sistemático sobre los fundamentos metodológicos y metafísicos de la teoría del espacio como intuición pura en la "Estética trascendental". Pamplona: Cuadernos de Anuario Filosófico, 2007.

Porfirio. "Porphyrii Isagoge et in Aristotelis Categorias commentarium". Commentaria in Aristotelem Graeca. IV. Berlín: Academia Litterarum Regia Borussica, 1887, pp. 1-22.

Posy, Carl, editor. Kant's Philosophy of Mathematics. Dordrecht: Kluwer, 1992.

Proclo. Procli Diadochi in primum Euclidis elementorum librum commentarii. Leipzig: Teubner, 1967.

Sellars, Wilfrid. Science and Metaphysics. Variations on kantian Themes. LondresNueva York: Routledge, 1968.

Simian, Rafael. "La existencia y la fundamentación de la metafísica". Ideas y Valores, vol. 60 , n. ${ }^{\circ} 147,2011$, pp. 113-41.

Smit, Houston. "Kant on Marks and the Inmediacy of Intuition". The Philosophical Review, vol. 109, n. ${ }^{\circ}$ 2, 2000, pp. 235-66. 
Tilliette, Xavier. Recherches sur l'intuition intellectuelle de Kant à Hegel. París: Vrin, 1995.

Torretti, Roberto. "Intuición pura". El sentimiento de lo humano en la ciencia, la filosofía y las artes. Editado por César Ojeda y Alejandro Ramírez. Santiago: Universitaria, 2004, pp. 111-34.

Willaschek, Marcus. "Phaenomena/Noumena und die Amphibolie der Reflexionsbegriffe". Immanuel Kant. Kritik der reinen Vernunft, Berlín: Akademie Verlag, 1998, pp. 325-51.

Willaschek, Markus, Jürgen Stolzenberg, Georg Mohr y Stefano Bacin, editores. Kant-Lexikon. Berlín-Boston: Walter de Gruyter, 2015.

Vigo, Alejandro. Estudios aristotélicos. Navarra: EUNSA, 2006.

Wilson, K. D. "Kant on Intuition". The Philosophical Quarterly, vol. 25, n. ${ }^{\circ}$ 100, 1975, pp. 247-65. 